\title{
Preparation and application of taste bud organoids in biomedicine towards chemical sensation mechanisms
}

\author{
Shuge Liu ${ }^{1}$, Ping Zhu ${ }^{2}$, Yulan Tian ${ }^{2}$, Yating Chen ${ }^{1}$, Yage Liu ${ }^{1}$, Wei Chen ${ }^{1}$, Liping Du ${ }^{2}$, and \\ Chunsheng $\mathrm{Wu}^{2}$ \\ ${ }^{1}$ Xi'an Jiaotong University Institute of Medical Engineering \\ ${ }^{2}$ Xi'an Jiaotong University
}

November 6, 2021

\begin{abstract}
Taste is one of the most basic and important sensations that is able to monitor the food quality and avoid intake of potential danger materials. Whether as an inevitable symptom of aging or a complication of cancer treatment, taste loss so seriously affects the patient's life quality. Taste bud organoids provide a great convenience for the research of taste functions and the underlying mechanisms due to their characteristics of availability, strong maneuverability, and high similarity to the in-vivo taste buds. This review gives a systemic and comprehensive introduction to the preparation and application of taste bud organoids towards chemical sensing mechanisms. For the first, the basic structure and function of taste buds in biomedicine will be brief introduced. Then, the currently available approaches for the preparation of taste bud organoids are summarized and discussed, which are mainly divided into two categories, i.e. stem/progenitor cell-derived approach and tissue-derived approach. For the next, different applications of taste bud organoids in biomedicine are outlined based on their central roles such as disease modeling, biological sensing, gene regulation, and signal transduction. Finally, the current challenges, future development trends and prospects of research in taste bud organoids are proposed and discussed.
\end{abstract}

\section{Introduction}

Taste buds are specialized organ for taste sensation, which are able to detect and report oral irritation caused by chemicals such as alcohol and capsaicin, thereby avoiding the intake of toxic and harmful foods. With the in-depth study of the coronavirus disease 2019 (COVID-19), in addition to common symptoms such as fever, cough, dyspnea, fatigue, and myalgia, loss of smell and taste are gradually being taken into consideration. A new study showed that up to $41 \%$ of people infected with severe acute respiratory syndrome coronavirus -2 (SARS-CoV-2) have a loss of taste[1]. At the same time, dysgeusia is very common in patients with head and neck cancer, and as many as $75 \%$ of patients complain of loss of taste[2]. Nearly $60 \%$ of patients receiving systemic chemotherapy will report a certain degree of dysgeusia, which is related to the type of cytotoxic drugs and the presence of oral mucositis[3]. A mount of factors such as cancer treatments, bacterial and viral illness, age and other medications may damage the taste system and reduce its function $[4,5]$.

Although tricyclic antidepressants[6], clonazepam or diazepam[7] have been proved to be useful for improving the abnormal sensation of taste, it's still lack effective treatment interventions. Most research on taste has focused at the cellular level, revealing the expression of receptors and the transmission of signals[8-10]. Meanwhile, since most of the mechanisms that cause dysgeusia are unclear, there is no clear treatment plan for dysgeusia. Therefore, in 2018, scientists made suggestions at a conference that potential therapies for dysgeusia could be provided by various approaches such as stem cell therapies, gene therapies, clinical research and patient engagement [11]. On the other hand, with the continuous deepening of research, animal models are gradually unable to meet the requirements of the experiment. For example, the immune rejection 
problem caused by organ transplantation, or the inconsistent test results caused by the difference between animal and human cells, and the test of drug efficacy and toxicity couldn't get more effective and true results. Copying and reconstructing human organs has become one of the directions that scientists focus on. Researchers have built taste bud organoids derived from the oral mucositis model mice to study the therapeutic effects of drugs on taste loss[12]. It is a model based on a 3D in-vitro cell culture system that is highly similar to the source tissues or organs in the body and widely used in cancer research[13]. Compared with cells, organoids have shown great advantages in many examples such as the earliest intestinal organoids[14], primary intestinal organoids in patients with cystic fibrosis[15], human pancreatic cancer organoids[16], liver cancer organoids[17], breast cancer organoids[18], and colorectal cancer organoids[19]. These tumor organoids show the same phenotype and disease characteristics as the original tumor tissues. They are used as models to study tumor occurrence and development, personalized medicine and new antitumor drug screening. Besides, organoids are also derived from normal tissues to study developmental problems and the mechanism of occurrence and development of diseases[20-23]. Within this context, there are relatively few studies focusing on taste bud organoids. However, significant progress and some interesting results have been achieved in the fields of taste bud organoids, which have attracted more and more attention. Therefore, through the establishment of taste bud organoids, taste transduction mechanisms or dysgeusia are capable of being studied more efficiently, which aims to achieve the purpose of restoring taste.

Animals must undergo the activation of TRP channels during the process of obtaining nutrients through food, so as to meet the needs of metabolism and growth and development. For example, TRPV1 gene have been associated with alterations in salty taste sensitivity and salt preference[24]. TRPV4 contributes to sour taste sensing[25]. TRPM5 has the ability to perceive bitterness, sweetness and umami[26,27]. Meanwhile, missing function of TRP channels have been associated with reduced ability to detect taste stimuli $[25,27,28]$. Bitter taste is regulated by 30 TAS2R bitter taste receptor genes[29]. The perception of sweetness and umami is realized by forming heteromers between TAS1R2 and TAS1R1 and TAS1R3, respectively[30]. Furthermore, Dias and colleagues found different genotypes and temperature have significant differences in sensitivity and preference to salty and sweetness taste[31,32]. Another study revealed the genetic basis of variation in taste perception between two populations with different lifestyle. The results showed there is a strong divergence in genes and transduction pathways of taste signals between two groups with different lifestyle[33]. Genes carry the genetic information of species, and the study of genes can fundamentally reveal the mechanism of diseases and promote the development of medicine. Therefore, using taste bud organoids to study taste preferences related genes can promote the development of this process faster.

This review gives a systemic and comprehensive introduction to the preparation and application of taste bud organoids towards chemical sensing mechanisms. For the first, the basic structure and function of taste buds will be brief introduced based on taste transduction pathways, i.e. the pathway of ion channels and $\mathrm{G}$ protein-coupled receptors (GPCR). Then two main types of taste receptors are introduced, i.e. T1Rs and T2Rs, which can identify taste molecules and transmit taste signals downstream individually or at dimers manner. At the same time, taste receptors are not only functioned in taste buds but also play roles in the intestines, airways, and brain. Therefore, in addition to sensing taste, taste receptors can also cause an immune response in the body to deal with inflammation. Most importantly, the appearance of taste organoids provides a very convenient tool for the study of taste functions. Drawing lessons from other 3D structures to replicate certain characteristics of tissues and organs, and to simulate the application of disease states, taste bud organoids can also be used in the fields of drug screening, toxicity detection, disease modeling, and regenerative medicine in the future, with broad development prospects. For the next, this review also summarizes the approaches for preparation of taste bud organoids from stem/ progenitor cells or tissue. These approaches provide a very valuable reference for the study of taste bud organoids, allowing more scientists to conduct more in-depth research on taste sensation. Then, the applications of other types of organoids in diseases and biosensors are summarized and discussed. This is also the direction of the future development of taste bud organoids. Finally, the limitations and challenges of the development of taste bud organoids are discussed, and future development trends have also been prospected.

\section{Taste sensation}


It is the taste cells located in the taste buds of oral epithelium that are responsible for the perception of taste. There are three types of taste cells, including glial-like cells (Type I), sweet/bitter/umami detectors (Type II), and sour receptor cells (Type III) (Figure 1a), which are mainly distributed in circumvallate (CV) papillae, foliate (FL) papillae, and fungiform (FF) papillae[34,35]. All three kinds of cells are derived from $\mathrm{K}^{+} / \mathrm{K}_{1} 4^{+}$cells, which form the precursors, $\mathrm{Shh}^{+}$basal cells, of all three taste cell types in low level of $\beta$-catenin[34] (Figure 1b) and an average taste cell lifespan of 8-12 days in mammalians [36] and 10-14 in rodents, although different types of taste cells showed different lifespan[37]. Some taste cells transmit taste stimuli through taste receptors and then generate second messengers while others allow the stimulus to be transported into the cytoplasm to directly activate downstream reactions[38]. The most studied is the type II taste receptors, which means that bitter, sweet, and umami tastes use GPCRs and second messenger signaling mechanisms to transmit taste signals. The sour and salty stimulus use ion channels for transduction $[39,40]$.

The information transmission pathway of each taste is different. The sense of salty taste is mainly completed by the specific sodium salt taste receptor epithelial sodium channel (epithelial sodium channel, ENaC) and the non-specific sodium salt taste receptor capsaicin receptor 1 (vanilloid receptor-1 nonselective cation channel 1, TRPV1). It is recognized on the front of the tongue. Sour taste is the taste sensation produced by $\mathrm{H}^{+}$entering the oral cavity to stimulate the tongue mucosa. Transient receptor potential (TRP) channel members polycystin kidney dominant 1 like 3 (PKD1L3) and PKD2L1 are participate in sour sensation at the back of the tongue. The sweet and umami taste receptors (T1R2/T1R3 and T1R1/T1R3 receptors) distributed in the tongue epithelium are coupled to the taste-specific G protein $\alpha$-gustducin present on the taste buds, releasing G $\beta \gamma$ subunits, and activating phospholipase C- $\beta 2$ (phospholipase C- $\beta 2$ ), PLC- $\beta 2$ ) and adenylyl cyclase (AC). This will activate the downstream inositol triphosphate (IP3) and cAMP pathways, and the intracellular $\mathrm{Ca}_{2}{ }^{+}$concentration increases, which in turn activates the transient receptor potential channel M5 (transient receptor potential channel M5, TRPM5) depolarizing the cell membrane and releasing neurotransmitters, thereby producing sweetness. There are differences in the intracellular signal transduction of sweet receptors at different sites. The bitter taste receptor proteins (T2Rs) are distributed in the root of the tongue and combines with the bitter substance dissolved in the liquid phase to activate the taste cells and depolarize the cell membrane. Then it causes the nerve cells to post-synaptic excitement and is transmitted to the bitter taste center of the cerebral cortex[38].

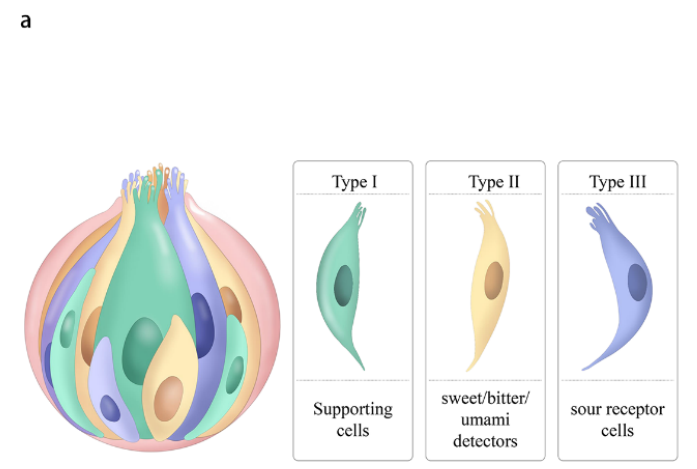

b

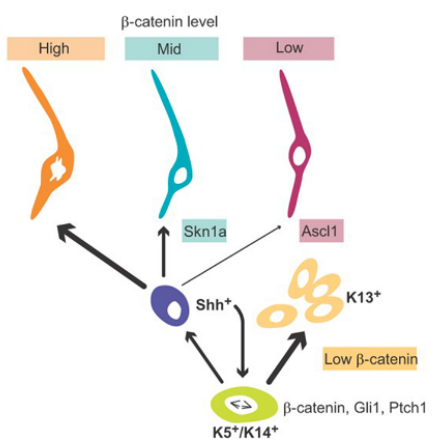

Figure 1. Taste cells in taste buds. (a) Schematic diagram of different cell types. (b) Stem cell renewal, Source: 34. Reproduced with permission of Development.

Taste receptors act as intermediates and interact with taste stimuli or ligands to activate afferent nerves and transmit taste signals to the brain. The taste receptor genes are expressed either in all types of taste buds or specific taste buds. In FF and FL papillae, T1R1 and T1R3 form dimers to sense umami. In CV, FL, and FF papillae, T1R2 and T1R3 are coexpressed to transfer sweetness[41-46]. Meanwhile, the main site of expression of the T2R genes is circumvallate taste buds sensing bitterness[47,48]. Salty and sour tastes are mediated by channel-type receptors[49,50]. And various candidate sour taste receptors have been discovered, such as ACCN1[51], HCN1, HCN4[52], TASK-1[53]. They can work alone or in combination with other 
receptors. Terms of the two best-studied families of taste receptors, T1R and T2R, have seven transmembrane characteristics and sometimes dimers are formed between them to play the role of transmitting taste signals (Figure 2).

Besides, taste receptors are also expressed in other tissues. In addition to being widely expressed in taste buds to sense bitterness, T2Rs may also be found in the intestine and human airway smooth muscle (ASM)[10] to detect toxic substances[54]. Meanwhile, in cystic fibrosis bronchial epithelial (CuFi-1), normal bronchial epithelial (NuLi-1), airway smooth muscle (ASM), pulmonary artery smooth muscle (PASM), mammary epithelial, brain cells[55], and breast cancer cells, TAS2Rs had specific expression pattern[56]. For example, TAS2R3, 4, 5, 10, 13, 19, and 50 transcripts expressed at moderate levels and TAS2R14 and TAS2R20 (or TASR49) at high levels in the various tissues analyzed. Moreover, taste receptors also perform many other functions. It's been reported that the T2R bitter receptor mediated the release of IL-25 from intestinal villi induced by Trichinella spiralis. This provides new ideas for the design of drugs against parasites[57]. This result preliminarily proves the role of bitter taste receptors in an immune response. Another evidence showed that quinine stimulated the airway's innate immune defense by increasing the frequency of cilia beating and stimulating the production of $\mathrm{NO}$ in a manner consistent with $\mathrm{T} 2 \mathrm{R}$ activation, thereby effectively alleviating chronic sinusitis[58]. Besides, T2R10 is expressed in pancreatic cancer tissues and various other cancer cell lines and regulated the chemotherapy resistance of tumor cells[59]. To study the function of receptors, it is inevitable to add exogenous ligands. TAS2R14 is the most widely regulated bitter taste receptor and is expressed in a variety of extraoral tissues. Therefore, more potent ligands are needed to study its function. The existing literature has designed a TAS2R14 agonist based on structure-based molecular modeling and experimental data. Furthermore, its effectiveness has been greatly improved[60]. For the most clearly studied bitter taste receptor, bitterDB contains more than 1,000 bitter taste molecules, while the related receptors are close to 800 [61]. More recently, based on this database, researchers have developed the main calculation method for predicting bitterness based on the chemical structure of the compound, which has greatly promoted the development of the pharmaceutical industry[62]. In summary, taste receptors not only transmit taste in taste buds, but are also expressed in the intestine, airway and brain, and perform important functions such as immune defense.

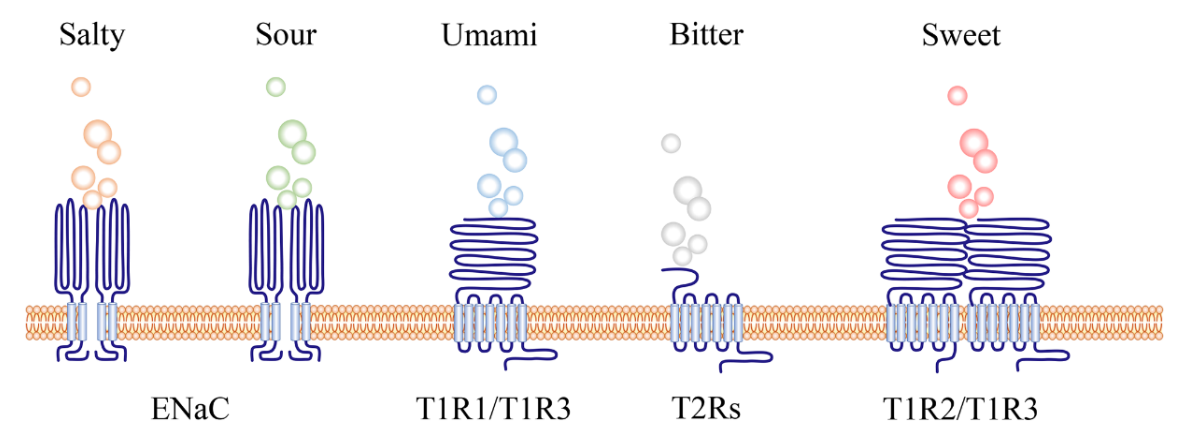

Figure 2. Schematic diagram of the transmembrane way and interaction of taste receptors.

\section{Preparation of taste bud organoids}

Many applications of human cancer models including cancer cell lines and patient-derived tumor xenografts (PDX) had emerged[13,63]. In these models, the primary tumor tissues were transplanted into immunodeficient mice[64,65]. PDX can better retain the complexity and heterogeneity of parental tumors, but it is still difficult to establish because of the high cost of analysis and long construction cycle. In recent years, patient-derived organoid (PDO) have made significant progress. The formation process of organoids is divided into self-mode events and morphogenesis rearrangements, which involve different mechanisms[66]. Any disturbance of physical or chemical signals may affect the acquisition of organoids. The earliest successful cultivation of taste organoids was based on fluorescence-activated cell sorting (FASC)[67]. Transgenic mice 
were used to screen out $\operatorname{Lgr} 5^{+}$cells, then taste bud was obtained ex vivo . It is proved that the obtained taste organoids contain proliferation and mature taste cells. Furthermore, the author used circumvallate papilla tissue to establish taste bud organoids[68]. Therefore, different preparation approaches can obtain taste bud organoids, but it seems that the tissue-derived approach is simpler and time-saving.

\subsection{Stem/progenitor cell-derived approach}

Taste bud organoids derived from stem cells only select stem/progenitor cells of taste cells for culture, thereby obtaining a more unitary culture system. Meanwhile, the stem/progenitor cell-derived culture method requires the labeling of taste progenitor cells in advance (Figure 3a). These cells are then sorted using FACS and cultured individually (Figure 3b-c). Finally, taste bud organoids can be achieved (Figure 3d). Inoculating taste cells on the sensor to simulate the perception of complex tastes to construct an artificial tongue model is the first utilization of taste cells to cultivate a tongue organ modelin vitro. The system detects tastants through high-efficiency functional receptors and provides ideas for artificial taste devices for taste perception and taste information standardization[9]. Moreover, in terms of disease treatment, taste bud organoids are able to mimic the state of the body well and produce consistent responses[69]. By transcriptome sequencing of organoids derived from $\mathrm{Lgr} 5^{+}$cells, preferential taste cell generation was age-related [70] and regulated via multiple signaling pathways[71], [72] which also enriches the signal transduction network that regulates the production of taste cells and provides a theoretical basis for the study of the proliferation and differentiation of taste cells as well as the development mechanism of taste buds.

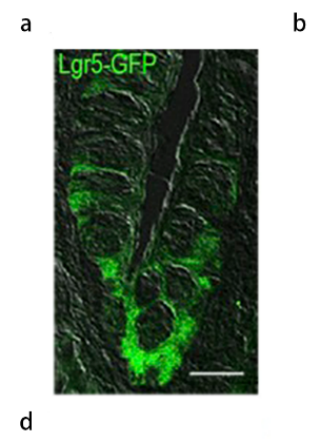

b
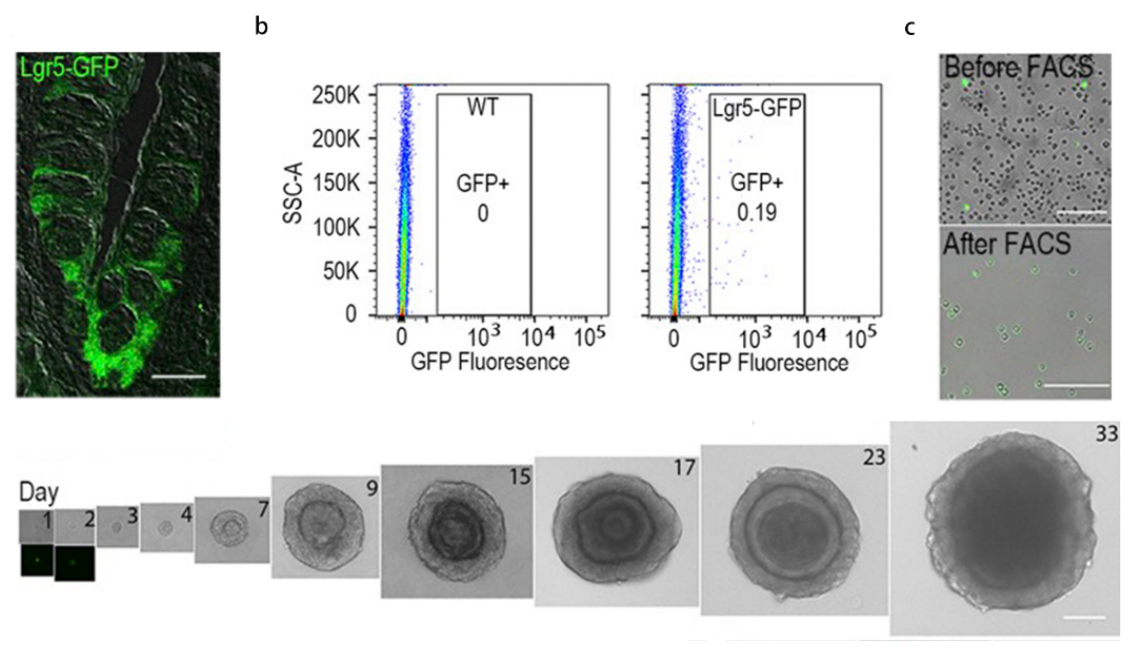

Figure 3. Lgr5+ cell-derived taste bud organoid culture. Source: 67. Reproduced with permission of Proc Natl Acad Sci USA. (a) Lgr $5^{+}$cells in the taste papilla. (b) Results of FACS sorting of Lgr $5^{+}$cells. (c) Single Lgr $5^{+}$cell (green). (d) Representative images of taste bud organoids at different days in culture.

\subsection{Tissue-derived approach}

The commonly used approach of cultivating taste bud organoids is to obtain the digestive juice of taste bud tissue and then cultivate it. Both mice and rats can be used as research objects to obtain taste buds for the cultivation of taste bud organoids. Injecting collagenase and dispase into the tongue to obtain the tongue epithelium, then separate the CV papillae, as well as part of the non-taste epithelium without taste buds[73]. [68] After further digesting the obtained taste papilla tissue with trypsin, it was mixed with matrigel and inoculated in a culture plate, and organoids were grown from these tissues (Figure 4). Then, the expression levels of taste bud lineage markers mRNA and proteins as well as immunofluorescence be used to characterize their physiological characteristics. The greatest convenience of the tissue-derived taste bud organoid culture approach is that there is no need to screen and culture specific cells and omit the step of single-cell sorting. In addition, the tissues contain other types of cells, which can better simulate the physiological conditions 
in the body and provide a living environment closer to the living body for the cultivation of organoids. Taste bud organoids from FUCCI2 mice in which mCherry-hCdt1 (red fluorescence) is expressed during G1 phase while mVenus-hGem (green fluorescence) is expressed during the S/G2/M phase of the cell cycle[68]. Another study showed that Trpv4 deficiency reduced sensitivity to sourness and the expression of type III cells in taste bud organoid[25]. These results can well prove that taste bud organoids derived from tissues can be used in research.

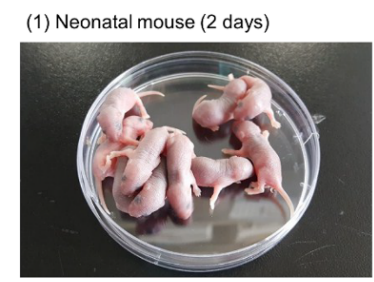

(4) Digest the tissues for $15 \mathrm{~min}$ and

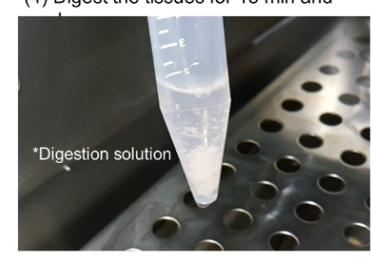

*Mixture of trypsin/EDTA,Accutase ${ }^{\oplus}$, and DNase I

(7) Sub-culture \#1 (Incubated for 2 days)

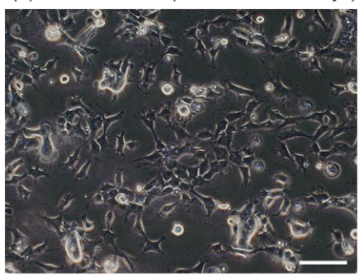

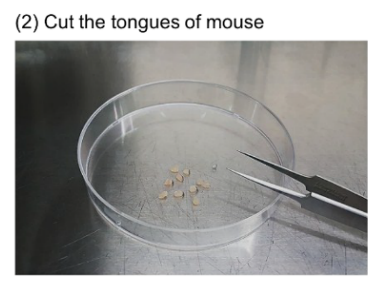

(5) Seed tissues to collagen I coated plate

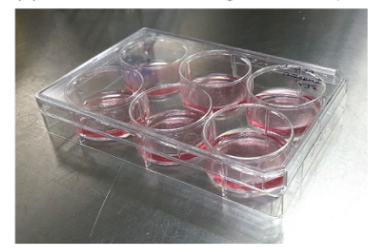

(8) Transfer to the experimental plate

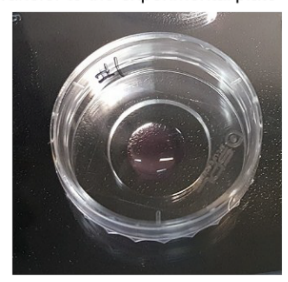

(3) Slice tongues in small tissues

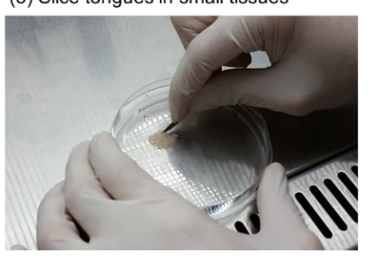

(6) Incubate the tissues for $4 \sim 5$ days

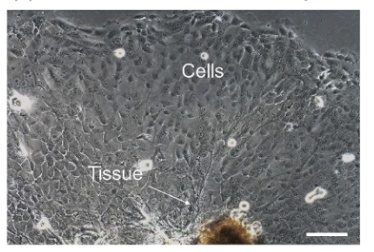

(9) Sub-culture \#2 (Incubate for 3 days)

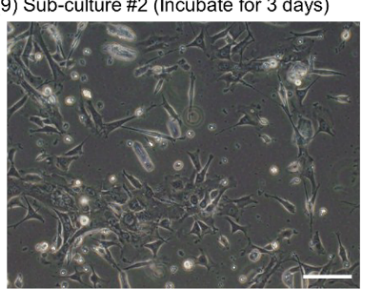

Figure 4. Tissue-derived taste bud organoid culture procedures. Source: 8. Reproduced with permission of Royal Society of Chemistry.

\section{Applications}

\subsection{Disease modeling}

The lack of suitable in-vitro models to accurately simulate specific tissues and disease states is an important factor restricting basic research and translational research. Organoids reproduce certain characteristics of tissues and organs, opening the door to a new world for disease research and drug development. As we all know, radiotherapy is a common method for cancer treatment, but there are many complications. In patients with head and neck cancer, radiation cause oral mucositis, which leads to loss of taste[74,75]. Radiation was given to taste bud organoids to construct a disease model of oral mucositis, and it was verified by a mouse experiment. The research showed that the results of organoids are consistent with that of the mouse test, that is, the SIRT1 inhibitor nicotinamide significantly relieved oral mucositis[12]. In previous clinical trials conducted in 2016, it was found that BIA 10-2474 (a fatty acid amide hydrolase inhibitor with the potential to treat a variety of neurological diseases) would produce neurotoxic accumulation in humans, but this situation did not occur in experimental animals. ChP organoids derived from human iPSCs also exhibit the toxicity accumulation of BIA 10-2474, indicating that this organoid model is more suitable for toxicity testing of new therapies than experimental animals[76].

Compared with the two-dimensional culture system, organoids help clarify the development, homeostasis, and pathogenesis of diseases and provide possible new approaches for the diagnosis and treatment of diseases. This organoid model was grown in a micro-machined chamber and was used for long-term in-situ imaging. It has 
been used to simulate cortical folding and study the pathogenesis of encephalopathy[77]. Studies have shown that brain organoids prepared from induced pluripotent stem cells of patients with lissencephaly have mitotic defects in the outer radial glial cells, which are rarely seen in mouse models[78]. Brain organoids are also used to study the Zika virus, which preferentially infects neural progenitor cells and reduces their proliferation and viability. This may be an important reason for the Zika virus to cause head deformities[79]. Talking about neural/stem cell biology, organoids could better track neurons and establish more predictivein-vitro disease models[80]. In regenerative medicine, artificial tissue cultivating was used to establish pathological mechanism research and transplantation research models[81]. Scientists combined with 3D culture organoid technology to develop a type 2 alveolar cell organoid model that could be cultured for a long time without a trophoblast for the development of vaccines and therapies[82]. Then, 3D self-rolled biosensor arrays (3DSR-BAs) of either active field-effect transistors or passive microelectrodes were implemented to interface human cardiac spheroids in 3D which enabled the development of organ-on-an-electronic-chip (organ-one-chip) platform[83] (Figure 5a-b). For taste sensing, bio-artificial tongue devices have been reported to sense comprehensive taste[9]. This is not only a device for taste detection but also provides an effective sensing device to simulate the function of the tongue outside the body, thereby providing a practical basis for exploring the mechanism of taste.

a
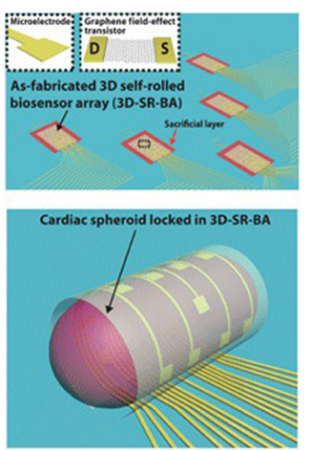
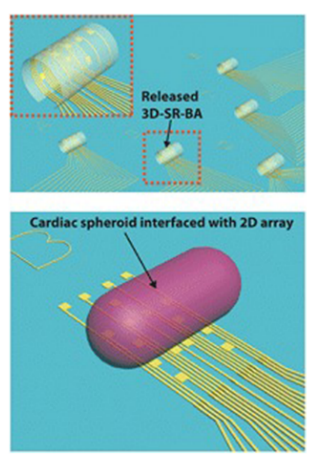

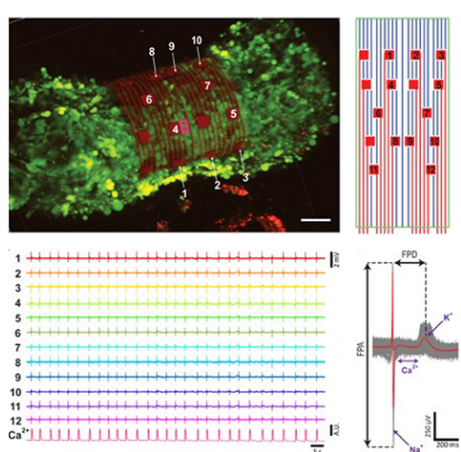

Figure 5. Organoid in three-dimensional self-rolled biosensor array. Source: 83. Reproduced with permission from Science Advances. (a) 3D-SR-BAs for electrical interrogation of human electrogenic spheroids. (b) Electrical recordings in 3D of cardiac spheroids.

\subsection{Biological sensing}

Microfluidics is a technology for precisely controlling and manipulating micro-scale fluids, especially submicron structures. It is also called Lab-on-a-Chip or microfluidic chip technology[84]. It integrates basic operation units such as sample preparation, reaction, separation, and detection in the biological, chemical, and medical analysis process on a micron-scale chip to automatically complete the entire analysis process. Due to its great potential in the fields of biology, chemistry, medicine, etc., it has developed into a new research field that intersects multiple disciplines such as biology, chemistry, medicine, fluids, electronics, materials, and machinery. At the same time, it also has the advantages of a lightweight, small amount of samples and reagents, low energy consumption, fast reaction speed, a large number of parallel processing, and ready-to-use disposables[85].

Suspended network technology has been fully utilized at present for the loading and cultivation of single-cell, multi-cell, and micro-tissue spheres. For example, Banerjee et al. used the hanging drop method to study the potential and cytotoxicity of stem cells to differentiate into specific lineages[86]. Another research used hanging-drop culture methods to distinguish the main erythroid progenitor cells derived from mouse tissue or human umbilical cord blood and compare with methylcellulose culture methods. The results proved that the behavior of isolated primary erythroid cells was comprehensively evaluated within the range of genetic and drug-induced disturbances[87]. For the application of embryonic bodies, the researchers used the 
differentiation of mouse embryonic stem cells (mESCs) to establish a model for evaluating embryotoxicity in vitro, and selected highly predictive protein markers specific to the developing heart tissue to enhance embryotoxicity, and then evaluated in vitro [88]. Multicellular tumorspheres (MCTS) are now widely used as organotypic models of normal and solid tumor tissues. Studies have used the hanging drop method to produce spheroids in the liver cancer cell line HepG2 breast adenocarcinoma cell line MCF-7, and it is a three-dimensional tissue-like structure that shows a high degree of the organization[89]. The use of 3D cell models in anticancer drug sensitivity testing is of great importance. The 384 hanging drop array plate has a high-throughput function and is used for cells with concentric layer patterning of different cell types and the cultivation of multiple cell types[90]. A compact on-chip pumping using the surface tension of the liquidair interface for flow drive realized the real-time feedback control loop of the beating of heart organoids derived from human induced pluripotent stem cells[91]. This method provides a way to study the effects of compounds on the heart itself and the effects of fluid circulation changes on other organ models in the system. There was also a device that seamlessly combines an open microfluidic device with fluorescenceactivated cell sorting (FACS), so that all cells, including stem cells, be directly sorted into the designated culture chamber in a fully automated manner and with high precision. Then, cells and microtissue culture were carried out in the form of hanging drops under controlled perfusion[92]. The potential for disease surveillance was also reported[93,94]. Furthermore, the corresponding inter-electrode impedance value could be calculated by reading the current generated by applying an $\mathrm{AC}$ voltage to evaluate the sample between the electrodes. Or through the chemical reaction to generate electrical current to monitor changes in material metabolism [94,95]. Meanwhile, the porous microfluidic platform is capable of multiplexing multiple drugs to a small biopsy tissue to evaluate the impact of drugs on its viability and microenvironment, thereby providing a testing platform for drug development and personalized medicine[96]. This method has been used for basic physiological research, metabolism, tumor biology, toxic alcohol, cell tissue, and bioartificial tissue development.

Therefore, it is speculated that the microfluidic hanging drop technology will also play a significant role in exploring the mechanism of taste loss or how to restore taste in patients with taste loss. Studies have shown that many regulatory factors play a role in the development of the taste system. Researchers have shown that artificial tongues sense smells more efficiently. This provides a reliable theoretical basis for the treatment of taste loss $[9,97,98]$.

The increasing progress in vitro models, chips integrating multiple organs have been made in recent years, and it is a major step forward in organ-on-a-chip technology[99] (Figure 6a). Functional drug screening on a complete tumor sample of a specific patient is a promising method for determining the best therapy for each patient. The response of a patient's cancerous tissue biopsy to a single drug (or drug combination) is highly dependent on the tumor microenvironment (TME), which includes a variety of resident and infiltrating host cells, secreted factors, and extracellular matrix proteins. Combining the microanatomy of tumor tissue with microfluidics can better protect the tissue microenvironment in the intact tissue for drug testing[100] (Figure 6b). Because the tissue is taken directly from the patient, it does not cause any major damage to the tumor microenvironment. This technology can capture a large amount of micro-tissue. However, such tumor spheres only retain a limited tumor microenvironment, which highlights the need for new highthroughput drug screening platforms. Therefore, the combination of microfluidic technology and organoids can accurately reproduce the interaction between drugs and tumors, which greatly promotes drug screening and disease treatment. 

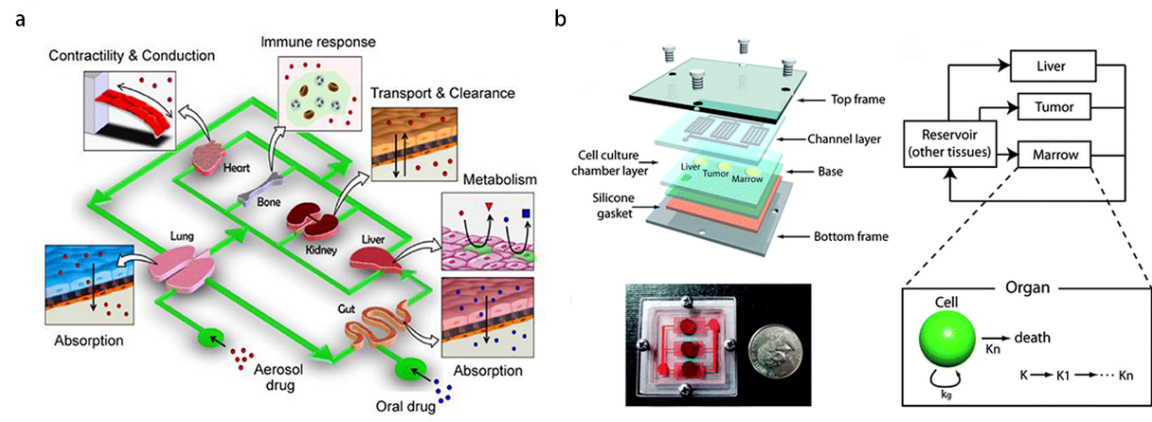

Figure 6. Schematic representation of organs-on-a-chip platform. (a) Organs-on-a-chip platforms. Source: 99. Reproduced with permission from Trends Cell Biol. (b) A microfluidic device with liver, tumor, and marrow. Source: 100. Reproduced with permission from Royal Society of Chemistry.

\subsection{Gene regulation}

The development of sequencing technology has also provided great convenience to the research of organoids. To understand the genes and signaling pathways involved in the development of taste buds, cDNAs were generated from organoids at different days, and performed RNA-Seq (RNA sequencing). The results showed that there are multiple signaling pathways and related genes during taste bud organoids development and different transcriptome landscape at the different growth stages (Figure 7a). Moreover, known transcripts of taste receptors were further clarified that appearing at late-stage organoids, and Tas2r126 was found as an emerging gene in taste bud development (Figure 7b)[71]. Then, the author obtained taste bud organoids derived from Lgr $5^{+}$cells of neonatal and adult mice. The confocal images showed neonatal mice generated more taste receptor cells than adult mice[70].

The taste system is regulated by many factors. Take Gli3, TRPV4, Sirt1, TNF, and IL-6 as examples. The members of the Glis family include Gli1,2,3 which are the key transcription factors of the Shh signaling pathway. They exist in two forms: activator and repressor. Hh blocked the inhibition of Smo activity by the twelve-times transmembrane protein Ptc, which reduce the production of the C-terminal truncated Gli repressor $\mathrm{Gli}^{\mathrm{R}}$. This promote the production of $\mathrm{Gli}$ activator $\mathrm{Gli}^{\mathrm{A}}$, and regulate the expression of target genes[101]. Because the regeneration of adult taste cells is affected by many factors such as aging, drug treatment, and various diseases. Gli3 is the main effector of Shh signaling pathway in adults. So, the researchers wanted to figure out what role Gli3 plays in the renewal of adult taste cells. A study has shown that Gli3 acted as a negative regulator to inhibit the proliferation of taste stem cells and the maturation of taste cells in taste bud organoids[97].

The Trp gene family encodes transient receptor potential (TRP) proteins which have different structures[102] and participates in a variety of physiological functions, affecting cell signal transduction[103]. In mammals, 28 Trp genes have been identified. Among them, TRP-melastatin 5 (TRPM5), polycystic kidney disease-1-like 3 (PKD1L3), polycystic kidney disease-2-like 1 (PKD2L1), and TRPV1t are expressed in taste cells[28,104]. The role of TRPM5 in taste perception has been well studied. In type II taste cells lacking synaptic connections, TRPM5 is activated by corresponding stimuli to generate action potentials, deliver ATP, and promote cell signal transduction[105-107]. However, TRPM5 is not the only ion channel that transmits bitter, sweet and umami tastes. TRPM4 has attracted considerable attention, both scholarly because its mRNA is present in taste cells[108] and its role in taste transduction has not been described[26]. Therefore, both TRPM4 and TRPM5 contribute to taste perception. Their absence will impair the sense of taste to a certain extent. PKD1L3 and PKD2L1 were co-expressed in taste receptor cells and form ion channels through interaction to realize taste perception[109,110]. And, TRPV1t regulates the perception of salty taste in the taste system[111]. A recent study related to TRPV4, another member of the TRP family, has suggested that TRPV4 realized the perception of sour taste by regulating the differentiation of type III taste cells[25]. 
The loss of taste is affected by many factors, such as aging and medication. In the aging process, the energy storage in the cell will be reduced, which will activate the sirtuins family and participate in the regulation of physiological processes. The sirtuins family includes seven members, which regulate energy metabolism through the protein lysine deacetylations. An updated study about sirt1 showed that SIRT1 inhibitors promoted $\mathrm{Lgr}^{+}$taste bud stem cell survival and mitigated radiation-induced oral mucositis in mice[12]. An initial study revealed that taste bud organoids had fast induction of TNF and IL-6 which was similar to native mouse taste epithelia[69]. In other words, taste buds have a unique mechanism to cope with inflammation. The epidermal growth factor (EGF) is a cell secretion factor that regulate the growth and development of hair and teeth. The first proof of the potential regulatory role of EGF in the fungal papilla model was reported in 2008. The author added human recombinant EGF for stimulation and found that EGF increased the number of fungal papillae and promoted the interpapillary tongue through PI3K/Akt, MEK/ERK, and p38 MAPK signals. The proliferation of epithelial cells prevents the rapid increase in the number of papillae induced by SHH destruction[112].

Genes are the basic genetic units that control biological traits. They produce proteins through translation to transmit information and control growth and development. Therefore, changes in the structure and function of genes will lead to many diseases. Gene therapy refers to the use of genetic engineering techniques to transfer normal genes into the cells of diseased patients to replace diseased genes, thereby expressing the lacking products, or by shutting down or reducing abnormally expressed genes, etc., to achieve certain treatment purpose of these genetic diseases. Using taste organoids to study the expression changes of important genes in the process of taste transmission undoubtedly provides a strong support for gene therapy. At the same time, it can speed up the research of taste transduction mechanism.

a

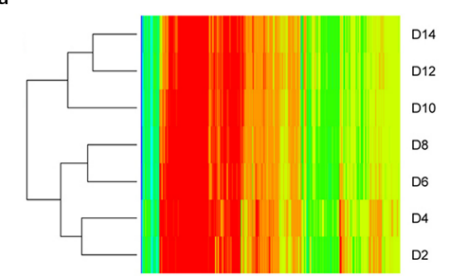

b

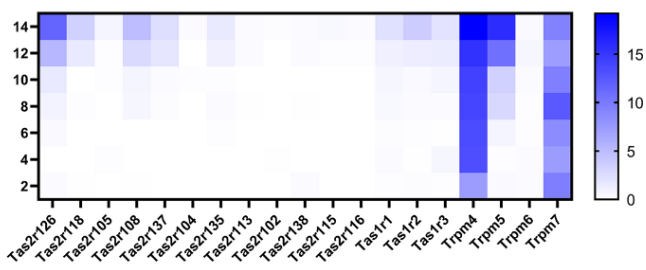

Figure 7. Genes related to taste system research. Source: 71. Reproduced with permission from Sci Rep. (a) The heat map of genes related to taste bud organoids development. (b) The heat map of major taste receptor genes.

\subsection{Signal transduction}

The development of taste is not only regulated by genes, but also by numerous signaling pathways, such as Wnt/ $\beta$-catenin[113], Shh[114], Fgf[115], Notch[116], and insulin[72] (Figure 8a-b). It has been proven that the Wnt- $\beta$-catenin signaling pathway initiated the development of taste papillae[113]. Therefore, Wnt signaling plays a vital role in the development of taste buds. Shh, receptor Ptc and downstream transcriptional activator Gli1 are abundantly expressed in tongue epithelial cells on the 12th day of embryonic stage and decreased in the expression on day 18[117]. In addition, after suppressing the Shh signal, the number of tongue papillae increased, the distance between tongue papillae reduced, and normal papillary development was destroyed[118]. Moreover, the expression of Shh in fungal papillae and the formation of normal mature fungal papillae depend on signal transduction through Wnt and $\beta$-catenin. Studies have shown that the activation of Wnt/ $\beta$-catenin signaling up-regulated the expression of Shh. In turn, blocking Shh signaling was accompanied by the upregulation of Wnt/ $\beta$-catenin signaling[119]. It was finally confirmed that Shh is an inhibitor of the Wnt/ $\beta$-catenin pathway. As early as 1999, fibroblast growth factors (FGFs) and their receptors had been detected in the developing tongue[120]. After knocking out Fgf10, the circumvallate papilla tissue of the mouse will disappear completely. But the effect on fungal papillae is reversed, that is, fungal papillae appear larger and more closely spaced. Therefore, the importance of the FGF signal transduction pathway 
in determining the number of CVP was determined[115]. The Notch signaling pathway has also been shown to be involved in the renewal of adult taste bud cells[121]. The authors used in situ hybridization to detect the expression of related genes in this pathway in both embryonic and adult mouse tongues. Insulin is a key molecule that regulates cell growth, energy metabolism, and synaptic plasticity. The insulin signaling pathway includes important regulatory factors such as PI3K, Akt, GSK-3ß, mTOR, MAPK, and GLUT[98]. Studies have verified that insulin plays an important role in taste cell differentiation/proliferation. There are a broad expression of IR and mTOR in mouse taste bud cells, including type I, II, III, and taste progenitor cells. Moreover, taste cell proliferation was significantly promoted after using rapamycin[72]. It is suggested that the insulin-mTOR signaling pathway may regulate the maintenance of taste bud homeostasis.

The organism is an organic whole, and any changes in molecules or substances will inevitably affect other components. Preliminary studies on these genes and pathways provide strong theoretical support for future exploration of the detailed mechanism of taste transmission, and accelerate the research of taste organoids to a certain extent.

a

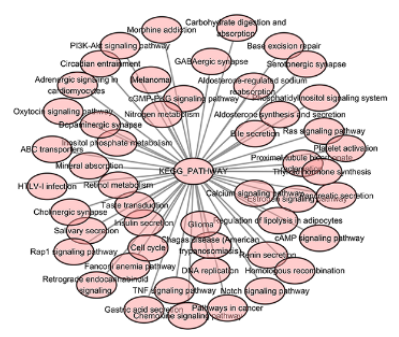

b

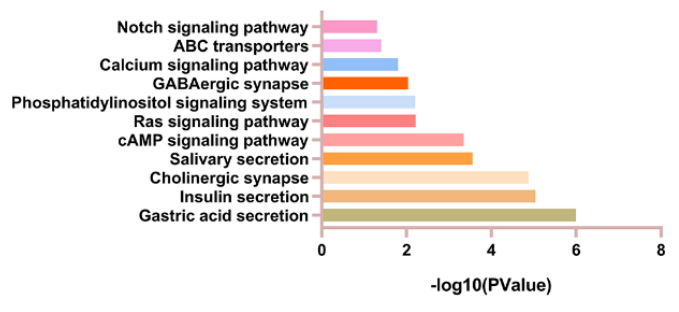

Figure 8. Signaling pathways related to taste system research. Source: 72. Reproduced with permission from PLoS One. (a) The KEGG pathway showed by Cytoscape. (b) Quantitative results of KEGG.

\section{Conclusions and Outlook}

Significant progress in the preparation and application of taste bud organoids has been achieved, which has promoted the research in the field of taste sensation. Specifically, various kinds of organoids have shown promising prospects and potential applications in many fields such as drug screening, toxicity testing, disease modeling, and regenerative medicine. The establishment of organoids is able to achieve more effective and true detection of drug efficacy and toxicity, precisely because organoids could be directly cultured and generated from human iPSCs, which largely avoids inconsistent test results caused by differences between animals and human cells. Therefore, this review systematically summarizes the existing research on taste bud organoids, laying a theoretical foundation for more in-depth research in this area in the future.

However, there are still many challenges for further development and application of taste bud organoids due to their own inherent shortcomings. For example, the construction of tissues and organsin vitro is still a big challenge. Therefore, it is hoped that the establishment and research of organoids are capable of furthering this challenge. Meanwhile, there are few applications for taste bud organoids. The current research using taste bud organoids reveals its proliferation ability, growth and development characteristics, oral mucositis treatment and a few functional genes researches, but the specific mechanism is still unclear. It is very likely that the research on organoids will mainly focus on disease models, such as development-related problems, genetic diseases, tumors, and cancers. By using the patient's iPSCs, valuable disease models have been established, which have been simulated and reproduced in vitro . However, in terms of taste bud organoids, there is few disease modeling, drug screening, or research combined with sensors. In spite of the first big step has been successfully taken, there are still many imperfections that need to be solved urgently, and the road to organoid research is continuing.

\section{Data Availability Statement}


The data that support the findings of this study are available from the corresponding author upon reasonable request.

\section{Declaration of competing interest}

There are no conflicts to declare.

Acknowledgments

This work was supported by grants from the National Natural Science Foundation of China (grant numbers 32071370, 51861145307, and 31700859), Natural Science Basic Research Plan in Shaanxi Province of China (grant number 2017JQ3037), and the Doctoral Fund of Education Ministry of China (grant number 2016M602832).

Reference

1. Qiu C., Cui C., Hautefort C., et al. (2020). Olfactory and Gustatory Dysfunction as an Early Identifier of COVID-19 in Adults and Children: An International Multicenter Study. Otolaryngol Head Neck Surg163 (4): 714-721. doi:10.1177/0194599820934376

2. Hovan A.J., Williams P.M., Stevenson-Moore P., et al. (2010). A systematic review of dysgeusia induced by cancer therapies.Support Care Cancer 18 (8): 1081-1087. doi:10.1007/s00520-010-0902-1

3. Okada N., Hanafusa T., Abe S., et al. (2016). Evaluation of the risk factors associated with high-dose chemotherapy-induced dysgeusia in patients undergoing autologous hematopoietic stem cell transplantation: possible usefulness of cryotherapy in dysgeusia prevention.Support Care Cancer 24 (9): 3979-3985. doi:10.1007/s00520-016-3244-9

4. Hummel T., Landis B.N., Hüttenbrink K.B. (2011). Smell and taste disorders. GMS Curr Top Otorhinolaryngol Head Neck Surg 10 Doc04. doi:10.3205/cto000077

5. Doty R.L. (2019). Treatments for smell and taste disorders: A critical review. Handb Clin Neurol 164 455-479. doi:10.1016/b978-0-444-63855-7.00025-3

6. Barker K.E., Batstone M.D., Savage N.W. (2009). Comparison of treatment modalities in burning mouth syndrome. Aust Dent J 54 (4): 300-305; quiz 396. doi:10.1111/j.1834-7819.2009.01154.x

7. Hampf G., Aalberg V., Sundén B. (1990). Experiences from a facial pain unit. J Craniomandib Disord 4 (4): 267-272.

8. Yun J., Cho A.N., Cho S.W., et al. (2018). DNA-mediated self-assembly of taste cells and neurons for taste signal transmission. Biomater Sci 6 (12): 3388-3396. doi:10.1039/c8bm00873f

9. Lee J.S., Cho A.N., Jin Y., et al. (2018). Bio-artificial tongue with tongue extracellular matrix and primary taste cells. Biomaterials151 24-37. doi:10.1016/j.biomaterials.2017.10.019

10. Deshpande D.A., Wang W.C., McIlmoyle E.L., et al. (2010). Bitter taste receptors on airway smooth muscle bronchodilate by localized calcium signaling and reverse obstruction. Nat Med 16 (11): 1299-1304. doi: $10.1038 / \mathrm{nm} .2237$

11. Mainland J.D., Barlow L.A., Munger S.D., et al. (2020). Identifying Treatments for Taste and Smell Disorders: Gaps and Opportunities.Chem Senses 45 (7): 493-502. doi:10.1093/chemse/bjaa038

12. Guo Q., Chen S., Rao X., et al. (2019). Inhibition of SIRT1 promotes taste bud stem cell survival and mitigates radiation-induced oral mucositis in mice. Am J Transl Res 11 (8): 4789-4799.

13. Drost J., Clevers H. (2018). Organoids in cancer research. Nat Rev Cancer 18 (7): $407-418$. doi:10.1038/s41568-018-0007-6

14. Barker N., van Es J.H., Kuipers J., et al. (2007). Identification of stem cells in small intestine and colon by marker gene Lgr5.Nature 449 (7165): 1003-1007. doi:10.1038/nature06196 
15. Dekkers J.F., Wiegerinck C.L., de Jonge H.R., et al. (2013). A functional CFTR assay using primary cystic fibrosis intestinal organoids. Nat Med 19 (7): 939-945. doi:10.1038/nm.3201

16. Boj S.F., Hwang C.I., Baker L.A., et al. (2015). Organoid models of human and mouse ductal pancreatic cancer. Cell 160 (1-2): 324-338. doi:10.1016/j.cell.2014.12.021

17. Broutier L., Mastrogiovanni G., Verstegen M.M., et al. (2017). Human primary liver cancer-derived organoid cultures for disease modeling and drug screening. Nat Med 23 (12): 1424-1435. doi:10.1038/nm.4438

18. Sachs N., de Ligt J., Kopper O., et al. (2018). A Living Biobank of Breast Cancer Organoids Captures Disease Heterogeneity. Cell 172 (1-2): 373-386.e310. doi:10.1016/j.cell.2017.11.010

19. van de Wetering M., Francies H.E., Francis J.M., et al. (2015). Prospective derivation of a living organoid biobank of colorectal cancer patients. Cell 161 (4): 933-945. doi:10.1016/j.cell.2015.03.053

20. Li X., Nadauld L., Ootani A., et al. (2014). Oncogenic transformation of diverse gastrointestinal tissues in primary organoid culture. Nat Med 20 (7): 769-777. doi:10.1038/nm.3585

21. Drost J., van Jaarsveld R.H., Ponsioen B., et al. (2015). Sequential cancer mutations in cultured human intestinal stem cells. Nature521 (7550): 43-47. doi:10.1038/nature14415

22. Matano M., Date S., Shimokawa M., et al. (2015). Modeling colorectal cancer using CRISPR-Cas9mediated engineering of human intestinal organoids. Nat Med 21 (3): 256-262. doi:10.1038/nm.3802

23. Drost J., van Boxtel R., Blokzijl F., et al. (2017). Use of CRISPR-modified human stem cell organoids to study the origin of mutational signatures in cancer. Science 358 (6360): 234-238. doi:10.1126/science.aao3130

24. Smith K.R., Treesukosol Y., Paedae A.B., et al. (2012). Contribution of the TRPV1 channel to salt taste quality in mice as assessed by conditioned taste aversion generalization and chorda tympani nerve responses. Am J Physiol Regul Integr Comp Physiol 303 (11): R1195-1205. doi:10.1152/ajpregu.00154.2012

25. Matsumoto K., Ohishi A., Iwatsuki K., et al. (2019). Transient receptor potential vanilloid 4 mediates sour taste sensing via type III taste cell differentiation. Sci Rep 9 (1): 6686. doi:10.1038/s41598-019-43254-y

26. Dutta Banik D., Martin L.E., Freichel M., et al. (2018). TRPM4 and TRPM5 are both required for normal signaling in taste receptor cells.Proc Natl Acad Sci U S A 115 (4): E772-e781. doi:10.1073/pnas.1718802115

27. Zhang Y., Hoon M.A., Chandrashekar J., et al. (2003). Coding of sweet, bitter, and umami tastes: different receptor cells sharing similar signaling pathways. Cell 112 (3): 293-301. doi:10.1016/s0092-8674(03)00071-0

28. Lyall V., Heck G.L., Vinnikova A.K., et al. (2004). The mammalian amiloride-insensitive non-specific salt taste receptor is a vanilloid receptor-1 variant. J Physiol 558 (Pt 1): 147-159. doi:10.1113/jphysiol.2004.065656

29. Behrens M., Meyerhof W. (2009). Mammalian bitter taste perception.Results Probl Cell Differ 47 203220. doi:10.1007/400_2008_5

30. Nelson G., Hoon M.A., Chandrashekar J., et al. (2001). Mammalian sweet taste receptors. Cell 106 (3): 381-390. doi:10.1016/s0092-8674(01)00451-2

31. Dias A.G., Rousseau D., Duizer L., et al. (2013). Genetic variation in putative salt taste receptors and salt taste perception in humans. Chem Senses 38 (2): 137-145. doi:10.1093/chemse/bjs090

32. Talavera K., Yasumatsu K., Voets T., et al. (2005). Heat activation of TRPM5 underlies thermal sensitivity of sweet taste. Nature 438 (7070): 1022-1025. doi:10.1038/nature04248

33. Sjöstrand A.E., Sjödin P., Hegay T., et al. (2021). Taste perception and lifestyle: insights from phenotype and genome data among Africans and Asians. Eur J Hum Genet 29 (2): 325-337. doi:10.1038/s41431-02000736-2 
34. Barlow L.A. (2015). Progress and renewal in gustation: new insights into taste bud development. Development 142 (21): 3620-3629. doi:10.1242/dev.120394

35. Barlow L.A., Klein O.D. (2015). Developing and regenerating a sense of taste. Curr Top Dev Biol 111 401-419. doi:10.1016/bs.ctdb.2014.11.012

36. Perea-Martinez I., Nagai T., Chaudhari N. (2013). Functional cell types in taste buds have distinct longevities. PLoS One 8 (1): e53399. doi:10.1371/journal.pone.0053399

37. Beidler L.M., Smallman R.L. (1965). Renewal of cells within taste buds. J Cell Biol 27 (2): 263-272. doi:10.1083/jcb.27.2.263

38. Roper S.D., Chaudhari N. (2017). Taste buds: cells, signals and synapses. Nat Rev Neurosci 18 (8): 485-497. doi:10.1038/nrn.2017.68

39. Kinnamon S.C. (2012). Taste receptor signalling - from tongues to lungs. Acta Physiol (Oxf) 204 (2): 158-168. doi:10.1111/j.1748-1716.2011.02308.x

40. Bachmanov A.A., Beauchamp G.K. (2007). Taste receptor genes.Annu Rev Nutr 27 389-414. doi:10.1146/annurev.nutr.26.061505.111329

41. Hoon M.A., Adler E., Lindemeier J., et al. (1999). Putative mammalian taste receptors: a class of tastespecific GPCRs with distinct topographic selectivity. Cell 96 (4): 541-551. doi:10.1016/s0092-8674(00)80658-3

42. Li X., Li W., Wang H., et al. (2005). Pseudogenization of a sweet-receptor gene accounts for cats' indifference toward sugar.PLoS Genet 1 (1): 27-35. doi:10.1371/journal.pgen.0010003

43. Damak S., Rong M., Yasumatsu K., et al. (2003). Detection of sweet and umami taste in the absence of taste receptor T1r3. Science301 (5634): 850-853. doi:10.1126/science.1087155

44. Max M., Shanker Y.G., Huang L., et al. (2001). Tas1r3, encoding a new candidate taste receptor, is allelic to the sweet responsiveness locus Sac. Nat Genet 28 (1): 58-63. doi:10.1038/ng0501-58

45. Montmayeur J.P., Liberles S.D., Matsunami H., et al. (2001). A candidate taste receptor gene near a sweet taste locus. Nat Neurosci 4 (5): 492-498. doi:10.1038/87440

46. Reed D.R., Li S., Li X., et al. (2004). Polymorphisms in the taste receptor gene (Tas1r3) region are associated with saccharin preference in 30 mouse strains. J Neurosci 24 (4): 938-946. doi:10.1523/jneurosci.137403.2004

47. Bufe B., Hofmann T., Krautwurst D., et al. (2002). The human TAS2R16 receptor mediates bitter taste in response to beta-glucopyranosides. Nat Genet 32 (3): 397-401. doi:10.1038/ng1014

48. Adler E., Hoon M.A., Mueller K.L., et al. (2000). A novel family of mammalian taste receptors. Cell 100 (6): 693-702. doi:10.1016/s0092-8674(00)80705-9

49. Desimone J.A., Ren Z., Phan T.H., et al. (2012). Changes in taste receptor cell [Ca2+]i modulate chorda tympani responses to salty and sour taste stimuli. J Neurophysiol 108 (12): 3206-3220. doi:10.1152/jn.00916.2011

50. Teng B., Wilson C.E., Tu Y.H., et al. (2019). Cellular and Neural Responses to Sour Stimuli Require the Proton Channel Otop1. Curr Biol 29 (21): 3647-3656.e3645. doi:10.1016/j.cub.2019.08.077

51. Ugawa S., Yamamoto T., Ueda T., et al. (2003). Amiloride-insensitive currents of the acid-sensing ion channel-2a (ASIC2a)/ASIC2b heteromeric sour-taste receptor channel. J Neurosci 23 (9): 3616-3622. doi:10.1523/jneurosci.23-09-03616.2003

52. Stevens D.R., Seifert R., Bufe B., et al. (2001). Hyperpolarization-activated channels HCN1 and HCN4 mediate responses to sour stimuli. Nature 413 (6856): 631-635. doi:10.1038/35098087 
53. Richter T.A., Dvoryanchikov G.A., Chaudhari N., et al. (2004). Acid-sensitive two-pore domain potassium (K2P) channels in mouse taste buds. J Neurophysiol 92 (3): 1928-1936. doi:10.1152/jn.00273.2004

54. Wu S.V., Rozengurt N., Yang M., et al. (2002). Expression of bitter taste receptors of the T2R family in the gastrointestinal tract and enteroendocrine STC-1 cells. Proc Natl Acad Sci U S A 99 (4): 2392-2397. doi:10.1073/pnas.042617699

55. Singh N., Vrontakis M., Parkinson F., et al. (2011). Functional bitter taste receptors are expressed in brain cells. Biochem Biophys Res Commun 406 (1): 146-151. doi:10.1016/j.bbrc.2011.02.016

56. Jaggupilli A., Singh N., Upadhyaya J., et al. (2017). Analysis of the expression of human bitter taste receptors in extraoral tissues. Mol Cell Biochem 426 (1-2): 137-147. doi:10.1007/s11010-016-2902-z

57. Luo X.C., Chen Z.H., Xue J.B., et al. (2019). Infection by the parasitic helminth Trichinella spiralis activates a Tas2r-mediated signaling pathway in intestinal tuft cells. Proc Natl Acad Sci U S A 116 (12): 5564-5569. doi:10.1073/pnas.1812901116

58. Workman A.D., Maina I.W., Brooks S.G., et al. (2018). The Role of Quinine-Responsive Taste Receptor Family 2 in Airway Immune Defense and Chronic Rhinosinusitis. Front Immunol 9624. doi:10.3389/fimmu.2018.00624

59. Stern L., Giese N., Hackert T., et al. (2018). Overcoming chemoresistance in pancreatic cancer cells: role of the bitter taste receptor T2R10. J Cancer 9 (4): 711-725. doi:10.7150/jca.21803

60. Di Pizio A., Waterloo L.A.W., Brox R., et al. (2020). Rational design of agonists for bitter taste receptor TAS2R14: from modeling to bench and back. Cell Mol Life Sci 77 (3): 531-542. doi:10.1007/s00018-01903194-2

61. Dagan-Wiener A., Di Pizio A., Nissim I., et al. (2019). BitterDB: taste ligands and receptors database in 2019. Nucleic Acids Res47 (D1): D1179-d1185. doi:10.1093/nar/gky974

62. Bahia M.S., Nissim I., Niv M.Y. (2018). Bitterness prediction in-silico: A step towards better drugs. Int J Pharm 536 (2): 526-529. doi:10.1016/j.ijpharm.2017.03.076

63. Schutgens F., Clevers H. (2020). Human Organoids: Tools for Understanding Biology and Treating Diseases. Annu Rev Pathol 15 211-234. doi:10.1146/annurev-pathmechdis-012419-032611

64. Seol H.S., Kang H.J., Lee S.I., et al. (2014). Development and characterization of a colon PDX model that reproduces drug responsiveness and the mutation profiles of its original tumor. Cancer Lett 345 (1): 56-64. doi:10.1016/j.canlet.2013.11.010

65. Zhu Y., Tian T., Li Z., et al. (2015). Establishment and characterization of patient-derived tumor xenograft using gastroscopic biopsies in gastric cancer. Sci Rep 5 8542. doi:10.1038/srep08542

66. Rossi G., Manfrin A., Lutolf M.P. (2018). Progress and potential in organoid research. Nat Rev Genet 19 (11): 671-687. doi:10.1038/s41576-018-0051-9

67. Ren W., Lewandowski B.C., Watson J., et al. (2014). Single Lgr5- or Lgr6-expressing taste stem/progenitor cells generate taste bud cells ex vivo. Proc Natl Acad Sci U S A 111 (46): 16401-16406. doi:10.1073/pnas.1409064111

68. Aihara E., Mahe M.M., Schumacher M.A., et al. (2015). Characterization of stem/progenitor cell cycle using murine circumvallate papilla taste bud organoid. Sci Rep 5 17185. doi:10.1038/srep17185

69. Feng S., Achoute L., Margolskee R.F., et al. (2020). Lipopolysaccharide-Induced Inflammatory Cytokine Expression in Taste Organoids. Chem Senses 45 (3): 187-194. doi:10.1093/chemse/bjaa002

70. Ren W., Liu Q., Zhang X., et al. (2020). Age-related taste cell generation in circumvallate papillae organoids via regulation of multiple signaling pathways. Exp Cell Res 394 (2): 112150. doi:10.1016/j.yexcr.2020.112150 
71. Ren W., Aihara E., Lei W., et al. (2017). Transcriptome analyses of taste organoids reveal multiple pathways involved in taste cell generation. Sci Rep 7 (1): 4004. doi:10.1038/s41598-017-04099-5

72. Takai S., Watanabe Y., Sanematsu K., et al. (2019). Effects of insulin signaling on mouse taste cell proliferation. PLoS One 14 (11): e0225190. doi:10.1371/journal.pone.0225190

73. Finger T.E., Danilova V., Barrows J., et al. (2005). ATP signaling is crucial for communication from taste buds to gustatory nerves.Science 310 (5753): 1495-1499. doi:10.1126/science.1118435

74. Nguyen H.M., Reyland M.E., Barlow L.A. (2012). Mechanisms of taste bud cell loss after head and neck irradiation. J Neurosci 32 (10): 3474-3484. doi:10.1523/jneurosci.4167-11.2012

75. Kawashita Y., Soutome S., Umeda M., et al. (2020). Oral management strategies for radiotherapy of head and neck cancer. Jpn Dent Sci Rev 56 (1): 62-67. doi:10.1016/j.jdsr.2020.02.001

76. Pellegrini L., Bonfio C., Chadwick J., et al. (2020). Human CNS barrier-forming organoids with cerebrospinal fluid production.Science 369 (6500). doi:10.1126/science.aaz5626

77. Di Donato N., Timms A.E., Aldinger K.A., et al. (2018). Analysis of 17 genes detects mutations in $81 \%$ of 811 patients with lissencephaly. Genet Med 20 (11): 1354-1364. doi:10.1038/gim.2018.8

78. Williams M., Prem S., Zhou X., et al. (2018). Rapid Detection of Neurodevelopmental Phenotypes in Human Neural Precursor Cells (NPCs).J Vis Exp (133). doi:10.3791/56628

79. Garcez P.P., Loiola E.C., Madeiro da Costa R., et al. (2016). Zika virus impairs growth in human neurospheres and brain organoids.Science 352 (6287): 816-818. doi:10.1126/science.aaf6116

80. Choi S.H., Kim Y.H., Hebisch M., et al. (2014). A three-dimensional human neural cell culture model of Alzheimer's disease. Nature515 (7526): 274-278. doi:10.1038/nature13800

81. Peng W.C., Logan C.Y., Fish M., et al. (2018). Inflammatory Cytokine TNF $\alpha$ Promotes the Long-Term Expansion of Primary Hepatocytes in 3D Culture. Cell 175 (6): 1607-1619.e1615. doi:10.1016/j.cell.2018.11.012

82. Youk J., Kim T., Evans K.V., et al. (2020). Three-Dimensional Human Alveolar Stem Cell Culture Models Reveal Infection Response to SARS-CoV-2. Cell Stem Cell 27 (6): 905-919.e910. doi:10.1016/j.stem.2020.10.004

83. Kalmykov A., Huang C., Bliley J., et al. (2019). Organ-on-e-chip: Three-dimensional self-rolled biosensor array for electrical interrogations of human electrogenic spheroids. Sci Adv 5 (8): eaax0729. doi:10.1126/sciadv.aax0729

84. Misun P.M., Birchler A.K., Lang M., et al. (2018). Fabrication and Operation of Microfluidic HangingDrop Networks. Methods Mol Biol1771 183-202. doi:10.1007/978-1-4939-7792-5_15

85. Mancera-Andrade E.I., Parsaeimehr A., Arevalo-Gallegos A., et al. (2018). Microfluidics technology for drug delivery: A review.Front Biosci (Elite Ed) 10 74-91. doi:10.2741/e809

86. Banerjee M., Bhonde R.R. (2006). Application of hanging drop technique for stem cell differentiation and cytotoxicity studies. Cytotechnology 51 (1): 1-5. doi:10.1007/s10616-006-9001-z

87. Gutiérrez L., Lindeboom F., Ferreira R., et al. (2005). A hanging drop culture method to study terminal erythroid differentiation.Exp Hematol 33 (10): 1083-1091. doi:10.1016/j.exphem.2005.06.014

88. Seiler A.E., Spielmann H. (2011). The validated embryonic stem cell test to predict embryotoxicity in vitro. Nat Protoc 6 (7): 961-978. doi:10.1038/nprot.2011.348

89. Kelm J.M., Timmins N.E., Brown C.J., et al. (2003). Method for generation of homogeneous multicellular tumor spheroids applicable to a wide variety of cell types. Biotechnol Bioeng 83 (2): 173-180. doi:10.1002/bit.10655 
90. Hsiao A.Y., Tung Y.C., Qu X., et al. (2012). 384 hanging drop arrays give excellent Z-factors and allow versatile formation of co-culture spheroids. Biotechnol Bioeng 109 (5): 1293-1304. doi:10.1002/bit.24399

91. Rismani Yazdi S., Shadmani A., Bürgel S.C., et al. (2015). Adding the 'heart' to hanging drop networks for microphysiological multi-tissue experiments. Lab Chip 15 (21): 4138-4147. doi:10.1039/c5lc01000d

92. Birchler A., Berger M., Jäggin V., et al. (2016). Seamless Combination of Fluorescence-Activated Cell Sorting and Hanging-Drop Networks for Individual Handling and Culturing of Stem Cells and Microtissue Spheroids. Anal Chem 88 (2): 1222-1229. doi:10.1021/acs.analchem.5b03513

93. Bartosh T.J., Ylöstalo J.H., Mohammadipoor A., et al. (2010). Aggregation of human mesenchymal stromal cells (MSCs) into 3D spheroids enhances their antiinflammatory properties. Proc Natl Acad Sci U S A 107 (31): 13724-13729. doi:10.1073/pnas.1008117107

94. Misun P.M., Rothe J., Schmid Y.R.F., et al. (2016). Multi-analyte biosensor interface for realtime monitoring of 3D microtissue spheroids in hanging-drop networks. Microsyst Nanoeng 216022. doi:10.1038/micronano.2016.22

95. Schmid Y.R.F., Bürgel S.C., Misun P.M., et al. (2016). Electrical Impedance Spectroscopy for Microtissue Spheroid Analysis in Hanging-Drop Networks. ACS Sensors 1 (8): 1028-1035. doi:10.1021/acssensors.6b00272

96. Horowitz L.F., Rodriguez A.D., Au-Yeung A., et al. (2021). Microdissected "cuboids" for microfluidic drug testing of intact tissues. Lab Chip 21 (1): 122-142. doi:10.1039/d0lc00801j

97. Qin Y., Sukumaran S.K., Jyotaki M., et al. (2018). Gli3 is a negative regulator of Tas1r3-expressing taste cells. PLoS Genet14 (2): e1007058. doi:10.1371/journal.pgen.1007058

98. Akhtar A., Sah S.P. (2020). Insulin signaling pathway and related molecules: Role in neurodegeneration and Alzheimer's disease.Neurochem Int 135 104707. doi:10.1016/j.neuint.2020.104707

99. Huh D., Hamilton G.A., Ingber D.E. (2011). From 3D cell culture to organs-on-chips. Trends Cell Biol 21 (12): 745-754. doi:10.1016/j.tcb.2011.09.005

100. Sung J.H., Kam C., Shuler M.L. (2010). A microfluidic device for a pharmacokinetic-pharmacodynamic (PK-PD) model on a chip. Lab Chip10 (4): 446-455. doi:10.1039/b917763a

101. Ingham P.W., McMahon A.P. (2001). Hedgehog signaling in animal development: paradigms and principles. Genes Dev 15 (23): 3059-3087. doi:10.1101/gad.938601

102. Veldhuis N.A., Poole D.P., Grace M., et al. (2015). The G protein-coupled receptor-transient receptor potential channel axis: molecular insights for targeting disorders of sensation and inflammation. Pharmacol Rev 67 (1): 36-73. doi:10.1124/pr.114.009555

103. Nilius B., Szallasi A. (2014). Transient receptor potential channels as drug targets: from the science of basic research to the art of medicine. Pharmacol Rev 66 (3): 676-814. doi:10.1124/pr.113.008268

104. Clapham D.E. (2003). TRP channels as cellular sensors.Nature 426 (6966): 517-524. doi:10.1038/nature02196

105. Wu H., Cui Y., He C., et al. (2020). Activation of the bitter taste sensor TRPM5 prevents high saltinduced cardiovascular dysfunction.Sci China Life Sci 63 (11): 1665-1677. doi:10.1007/s11427-019-1649-9

106. Pérez C.A., Huang L., Rong M., et al. (2002). A transient receptor potential channel expressed in taste receptor cells. Nat Neurosci5 (11): 1169-1176. doi:10.1038/nn952

107. Hofmann T., Chubanov V., Gudermann T., et al. (2003). TRPM5 is a voltage-modulated and $\mathrm{Ca}(2+)$-activated monovalent selective cation channel. Curr Biol 13 (13): 1153-1158. doi:10.1016/s09609822(03)00431-7 
108. Liu P., Shah B.P., Croasdell S., et al. (2011). Transient receptor potential channel type M5 is essential for fat taste. J Neurosci31 (23): 8634-8642. doi:10.1523/jneurosci.6273-10.2011

109. LopezJimenez N.D., Cavenagh M.M., Sainz E., et al. (2006). Two members of the TRPP family of ion channels, Pkd113 and Pkd211, are co-expressed in a subset of taste receptor cells. J Neurochem 98 (1): 68-77. doi:10.1111/j.1471-4159.2006.03842.x

110. Ishimaru Y., Katano Y., Yamamoto K., et al. (2010). Interaction between PKD1L3 and PKD2L1 through their transmembrane domains is required for localization of PKD2L1 at taste pores in taste cells of circumvallate and foliate papillae. Faseb $j 24$ (10): 4058-4067. doi:10.1096/fj.10-162925

111. Lyall V., Phan T.H., Ren Z., et al. (2010). Regulation of the putative TRPV1t salt taste receptor by phosphatidylinositol 4,5-bisphosphate. J Neurophysiol 103 (3): 1337-1349. doi:10.1152/jn.00883.2009

112. Liu H.X., Henson B.S., Zhou Y., et al. (2008). Fungiform papilla pattern: EGF regulates inter-papilla lingual epithelium and decreases papilla number by means of PI3K/Akt, MEK/ERK, and p38 MAPK signaling.Dev Dyn 237 (9): 2378-2393. doi:10.1002/dvdy.21657

113. Liu F., Thirumangalathu S., Gallant N.M., et al. (2007). Wnt-beta-catenin signaling initiates taste papilla development. Nat Genet 39 (1): 106-112. doi:10.1038/ng1932

114. Bitgood M.J., McMahon A.P. (1995). Hedgehog and Bmp genes are coexpressed at many diverse sites of cell-cell interaction in the mouse embryo. Dev Biol 172 (1): 126-138. doi:10.1006/dbio.1995.0010

115. Petersen C.I., Jheon A.H., Mostowfi P., et al. (2011). FGF signaling regulates the number of posterior taste papillae by controlling progenitor field size. PLoS Genet 7 (6): e1002098. doi:10.1371/journal.pgen.1002098

116. Seta Y., Seta C., Barlow L.A. (2003). Notch-associated gene expression in embryonic and adult taste papillae and taste buds suggests a role in taste cell lineage decisions. J Comp Neurol 464 (1): 49-61. doi:10.1002/cne.10787

117. Hall J.M., Hooper J.E., Finger T.E. (1999). Expression of sonic hedgehog, patched, and Gli1 in developing taste papillae of the mouse.J Comp Neurol 406 (2): 143-155. doi:10.1002/(sici)10969861(19990405)406:2<143::aid-cne1>3.0.co;2-x

118. Hall J.M., Bell M.L., Finger T.E. (2003). Disruption of sonic hedgehog signaling alters growth and patterning of lingual taste papillae. Dev Biol 255 (2): 263-277. doi:10.1016/s0012-1606(02)00048-9

119. Iwatsuki K., Liu H.X., Grónder A., et al. (2007). Wnt signaling interacts with Shh to regulate taste papilla development. Proc Natl Acad Sci U S A 104 (7): 2253-2258. doi:10.1073/pnas.0607399104

120. Jung H.S., Oropeza V., Thesleff I. (1999). Shh, Bmp-2, Bmp-4 and Fgf-8 are associated with initiation and patterning of mouse tongue papillae. Mech Dev 81 (1-2): 179-182. doi:10.1016/s0925-4773(98)00234-2

121. Seta Y., Toyono T., Kataoka S., et al. (2005). Regulation of taste bud cell differentiation by notch signaling pathway. Chem Senses30 Suppl 1 i48-49. doi:10.1093/chemse/bjh107

a

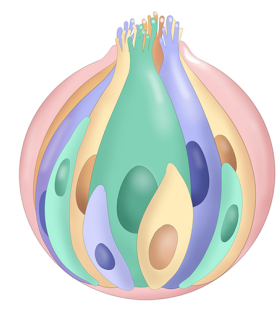

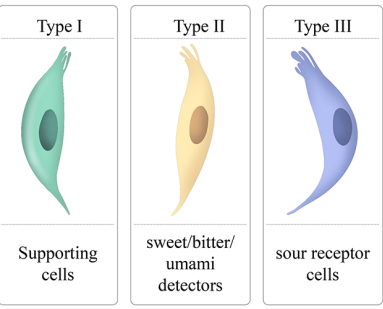

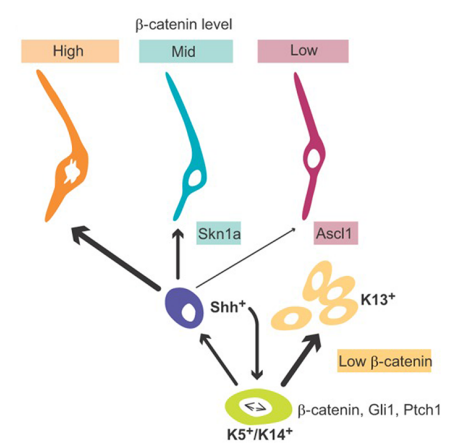




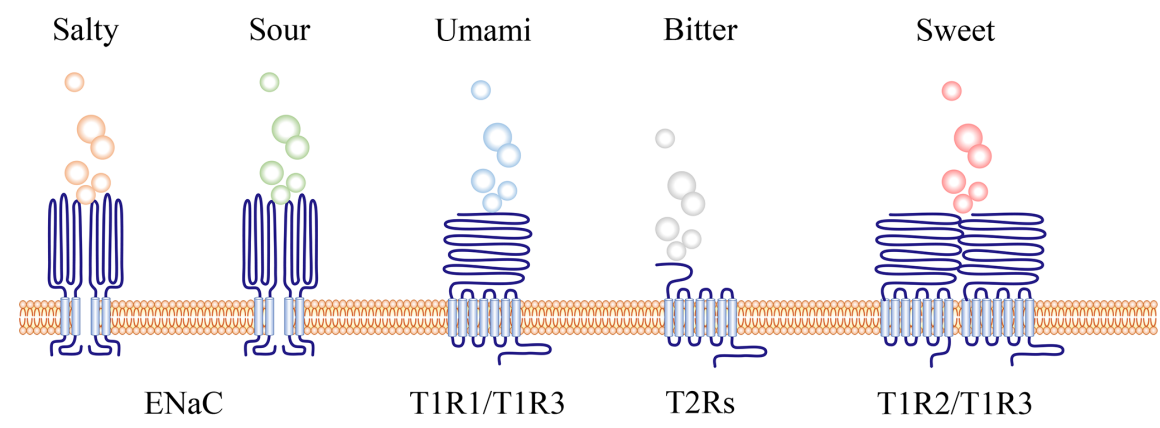

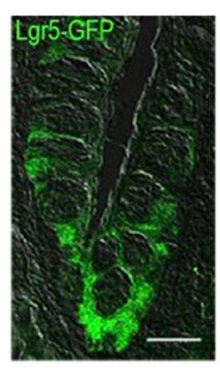

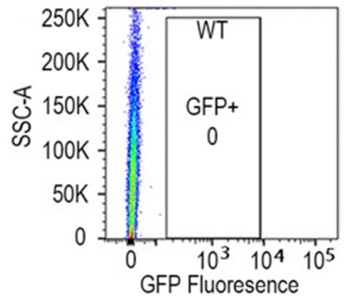

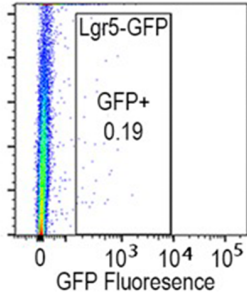

Before FAGs:
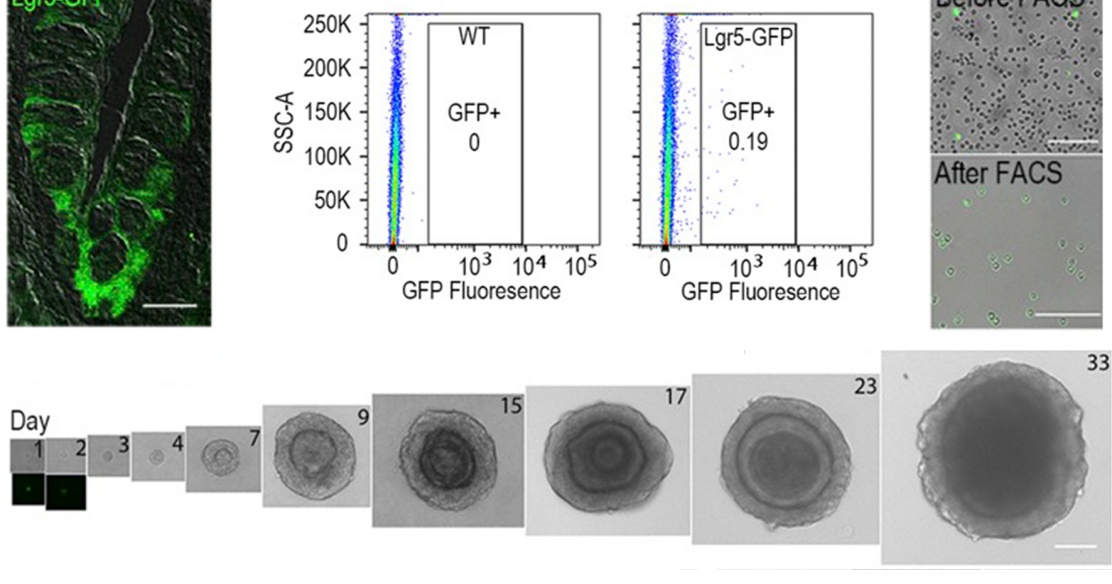

(1) Neonatal mouse (2 days)

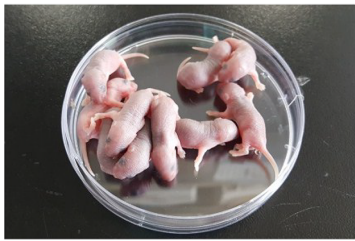

(4) Digest the tissues for $15 \mathrm{~min}$ and

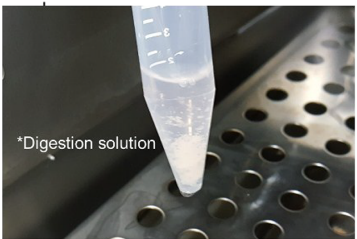

*Mixture of trypsin/EDTA, Accutases , and DNase I

(7) Sub-culture \#1 (Incubated for 2 days)

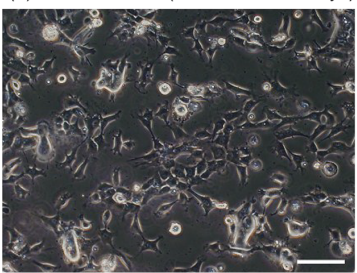

(2) Cut the tongues of mouse

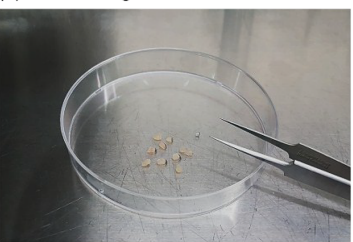

(5) Seed tissues to collagen I coated plate (6) Incubate the tissues for $4 \sim 5$ days

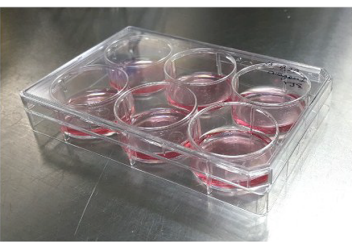

(8) Transfer to the experimental plate

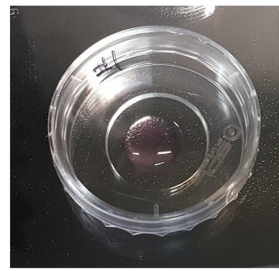

(3) Slice tongues in small tissues
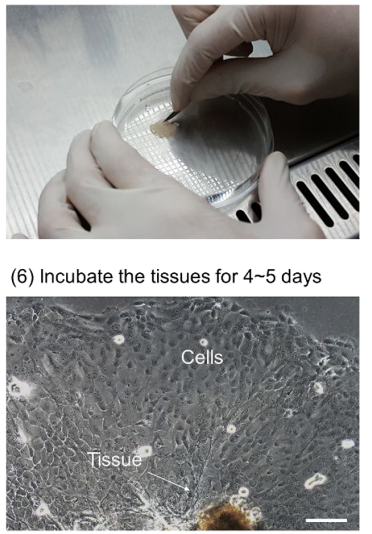

(9) Sub-culture \#2 (Incubate for 3 days)

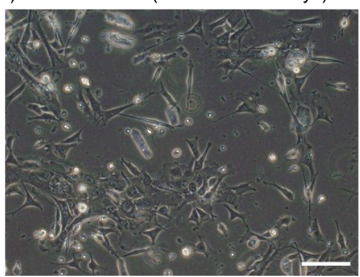


a

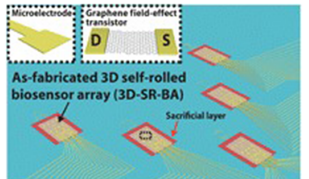

Cardiac spheroid locked in 3D-SR-BA
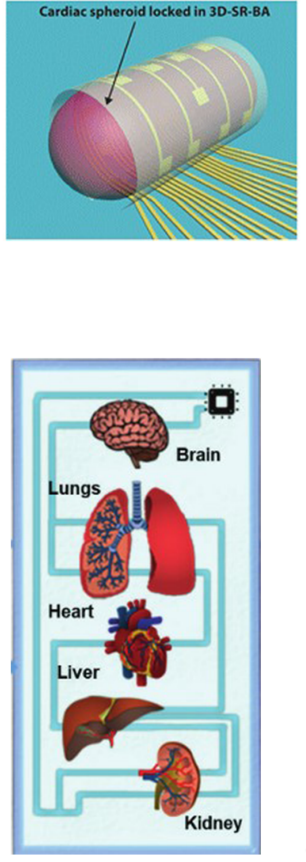

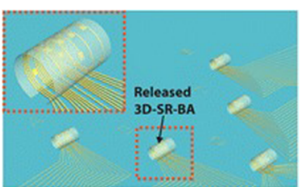

Cardiac spheroid interfaced with 20 array

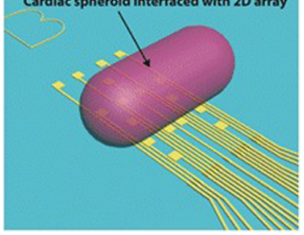

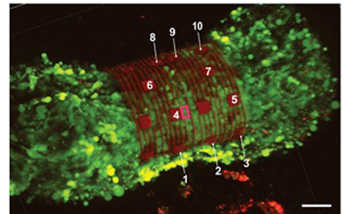
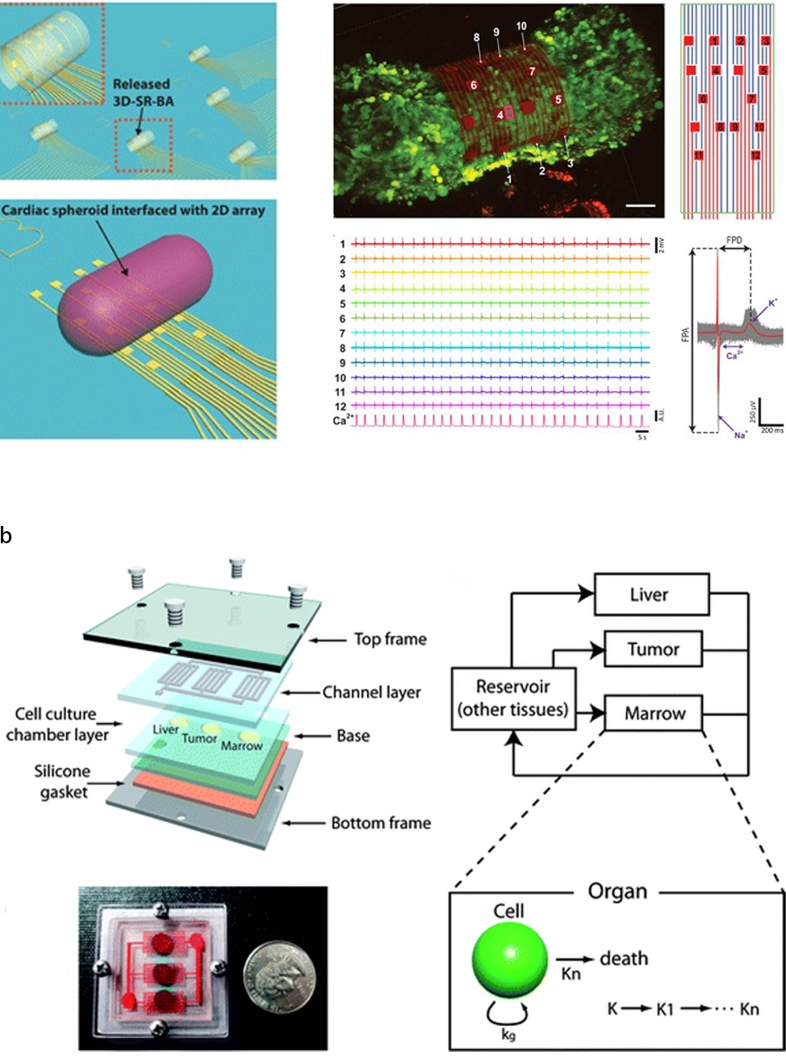
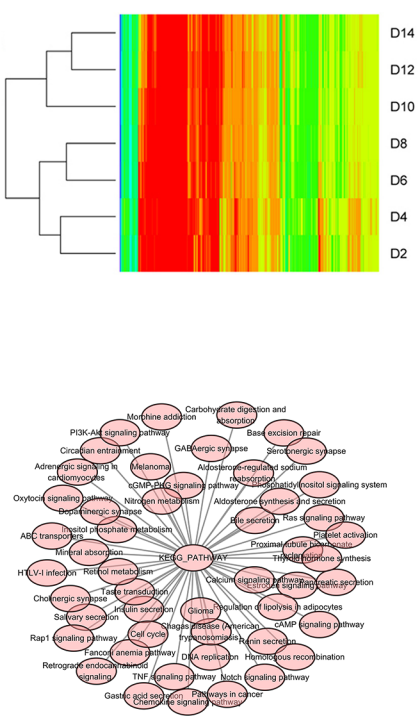

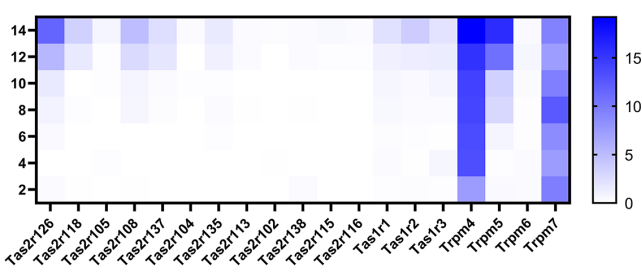

b

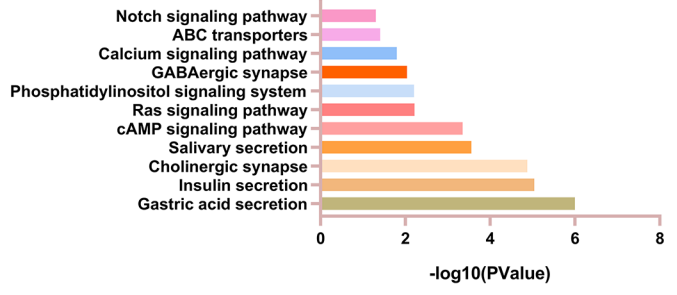

